\title{
FINAL STATE INTERACTION IN INCLUSIVE AND EXCLUSIVE QUASI-ELASTIC PROCESSES
}

\author{
M. ALVIOLI ${ }^{1}$, M.A. BRAUN ${ }^{2}$, C. CIOFI DEGLI ATTI ${ }^{1}$, L.P. KAPTARI ${ }^{3}$, \\ H. MORITA ${ }^{4}$, D. TRELEANI ${ }^{5}$ \\ (The Dubna-Hokkaido-St.Petersburg-Perugia-Trieste collaboration) \\ ${ }^{1}$ Department of Physics, University of Perugia, and Istituto Nazionale di Fisica \\ Nucleare, Sezione di Perugia, Via A. Pascoli, I-06100 Perugia, Italy \\ ${ }^{2}$ Department of High-Energy Physics, S.Petersburg University, 198904 \\ S.Petersburg, Russia \\ ${ }^{3}$ Bogoliubov Laboratory of Theoretical Physics, Joint Institute for Nuclear \\ Research, Dubna, Russia \\ ${ }^{4}$ Sapporo Gakuin University, Bunkyo-dai 11, Ebetsu 069, Hokkaido, Japan \\ ${ }^{5}$ Department of Theoretical Physics, University of Trieste, Strada Costiera 11, \\ Istituto Nazionale di Fisica Nucleare, Sezione di Trieste, and ICTP, I-34014, \\ Trieste, Italy
}

\begin{abstract}
We discuss a new approach to final state interactions, that keeps explicitly into account the virtuality of the ejected nucleon in quasi-elastic $A\left(e, e^{\prime} p\right) X$ scattering at very large $Q^{2}$, and we present some recent results, at moderately large $Q^{2}$ values, for the nuclear transparency in ${ }^{4} \mathrm{He},{ }^{16} \mathrm{O}$ and ${ }^{40} \mathrm{Ca}$ and for the momentum distributions of ${ }^{4} \mathrm{He}$.
\end{abstract}

\section{Finite formation time in quasi-elastic $A\left(e, e^{\prime}\right) X$ processes}

Final state interactions in inclusive quasi-elastic $A\left(e, e^{\prime}\right) X$ processes at large $Q^{2}$ are characterized by the large virtuality of the ejected nucleon. A straightforward way to incorporate virtuality effects in the process is through the Feynman diagrams formalism[1]. The amplitude describing $n$ consecutive rescattering of the ejectile emerging from the interaction of the struck nucleon with the incoming virtual photon, is represented in the diagram in Fig.1. The Glauber expression of the FSI may be obtained from the amplitude of the process in momentum space, obtained by the diagram, by applying the following procedure: one should first take the residues of the target nucleons propagators, to make the energy integrals of the loop variables. Then, while taking the Fourier transform to go to the coordinates representation, one should make the further approximations of $i$ ) disregarding the propagators of the ejected

cortona1: submitted to World Scientific on November 19, 2018 


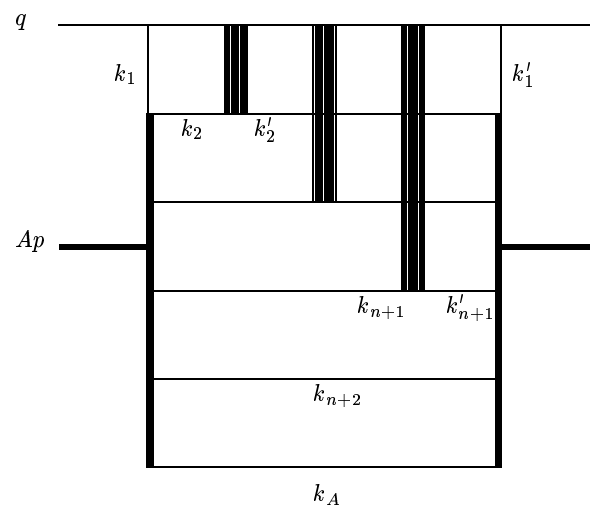

Figure 1. The forward scattering amplitude.

nucleon in the transverse momentum integrals, and $i i$ ) of keeping into account only the propagators of the ejected nucleon when making the longitudinal momentum integrations. As a result, the amplitude is expressed as an integral over the transverse coordinate $b$ of the struck nucleon, with respect to the direction of the virtual photon, and on the longitudinal coordinates of the interaction points $z_{i}, i=1,2, \ldots n+2$, with the appropriate nuclear density matrices.

To introduce the dependence on the virtualities $v_{j}=\left(k_{1}^{(j)}\right)^{2}-m^{2}$, the simplest assumption on the nucleon-nucleon amplitude $f_{j}$, is to use the factorized form[2]

$$
f_{j}=F\left(v_{j-1}\right) F\left(v_{j}\right) f ; \quad \gamma\left(k_{1}, q\right)=F\left(v_{1}\right) \gamma\left(Q^{2}\right)
$$

where $f$ is the on-shell amplitude and $F(v)$ a form-factor exhibiting the dependence of $f$ on the virtuality of the external lines, normalized according to $F(0)=1$ and decreasing with $v$. It is natural to introduce the dependence of the off-mass-shell electric form-factor $\gamma$ on the virtuality of the struck nucleon in the same manner. Going to the coordinates representation, using the recipe described above, one obtains in this way that all integrations on the longitudinal momenta become factorized. Each term in the product has the form

$$
i J(-z)=\int \frac{d v}{2 \pi} \frac{F^{2}(v)}{-v-i 0} \exp \left(i \frac{x m}{Q^{2}} v z\right)
$$

so that, if the dependence on the virtuality of the nucleon-nucleon interaction amplitude is disregarded (namely for $F(v)=1$ ), one obtains $J(z) \rightarrow \theta(z)$. 
The introduction of the dependence of the amplitudes on the virtualities, by means of the factorized expression (1), is therefore effectively equivalent to the replacement of the usual $\theta(z)$ in the nucleon propagator with the function $J(z)$, which depends on the virtuality through the form-factor $F(v)$.

On rather general grounds one may express the form-factor squared as

$$
F^{2}(v)=\int_{0}^{+\infty} \frac{d v^{\prime} v^{\prime} \tau\left(v^{\prime}\right)}{v^{\prime}-v-i 0} ; \quad \int_{0}^{+\infty} d v \tau(v)=1
$$

so that one may write

$$
J(z)=\theta(z) \int_{0}^{+\infty} d v \tau(v)\left(1-\exp \left(-i \frac{x m v z}{Q^{2}}\right)\right)
$$

and, with the simplest choice $\tau(v)=\delta\left(v-M^{2}\right)$, one obtains

$$
J(z)=\theta(z)\left[1-\exp \left(-i \frac{z}{l\left(Q^{2}\right)}\right)\right]
$$

where $l\left(Q^{2}\right)=Q^{2} /\left(x m M^{2}\right)$ has the obvious meaning of a formation length growing linearly with $Q^{2}$.

At the level of a single rescattering, the picture of FSI obtained in this way coincides with a standard two-channel Glauber model for the propagating nucleon and its excited state of mass squared $m^{* 2}=m^{2}+M^{2}$. Indeed the amplitude with a single rescattering is written as

$$
\mathcal{A}^{(1)}=\left\langle\gamma^{2} f\left[1-e^{-i\left(z_{1}^{\prime}-z\right) / l}-e^{-i\left(z-z_{1}\right) / l}+e^{-i\left(z_{1}^{\prime}-z_{1}\right) / l}\right]\right\rangle_{1}
$$

On the other hand, the two-channel Glauber model with two ejectile states 1 (the nucleon) and 2 (its excited state) leads to the single rescattering contribution

$\mathcal{A}^{(1)}=\left\langle\gamma_{1}^{2} f_{11}+\gamma_{1} \gamma_{2} f_{21} e^{-i\left(z_{1}^{\prime}-z\right) / l}+\gamma_{1} \gamma_{2} f_{12} e^{-i\left(z-z_{1}\right) / l}+\gamma_{2}^{2} f_{22} e^{-i\left(z_{1}^{\prime}-z_{1}\right) / l}\right\rangle_{1}$

where the average $\langle\ldots\rangle$ is defined as

$$
\begin{aligned}
\left\langle\mathcal{O}\left(b_{1}, z, z_{1}, z_{1}^{\prime}\right)\right\rangle_{1} \equiv- & \frac{x^{2} m}{2} A(A-1) \int d^{2} b_{1} d z d z_{1} d z_{1}^{\prime} \mathcal{O}\left(b_{1}, z, z_{1}, z_{1}^{\prime}\right) \\
& \times \rho\left(b_{1}, z\right) \rho\left(b_{1} z_{1} \mid b_{1} z_{1}^{\prime}\right) \theta\left(z_{1}^{\prime}-z\right) \theta\left(z-z_{1}\right) e^{i \Delta\left(z_{1}^{\prime}-z_{1}\right)}
\end{aligned}
$$

with $\Delta=Q^{2}(1-x) /\left(2 q_{z} x\right), \rho$ the density matrix, $f_{i k}=f_{k i}, i, k=1,2$ the forward scattering amplitudes for transitions $i \rightarrow k$ and $\gamma_{i}, i=1,2$ the vertices for the production of the two ejectile states. One immediately observes that (6) and (7) coincide if

$$
f_{11} \gamma_{1}+f_{12} \gamma_{2}=0, \quad f_{21} \gamma_{1}+f_{22} \gamma_{2}=0
$$

cortona1: submitted to World Scientific on November 19, 2018 
and, moreover, if $\gamma_{11}^{2} f_{11}$ in (7) is identified with $\gamma^{2} f$ in (6). The meaning of the sum rules (8) is that when applying the matrix $f_{i k}$ to the vector $\gamma_{i}$ one obtains zero, which, as discussed in ref.[3], is precisely the condition for color transparency. In the case of two channels one may easily see that both unitarity, $2 \Im \mathrm{m} f_{i l}=\sum_{j=1,2} f_{i j} f_{j l}^{*}$, and the transparency conditions are satisfied by

$$
f_{12}=f_{21}=-\xi f_{11}, \quad f_{22}=\xi^{2} f_{11}
$$

where $\xi$ is the (real) ratio of the form factors $\gamma_{1}$ and $\gamma_{2}$, whose value is obtained by $\xi^{2}=\left|f_{12}\right|^{2} /\left|f_{11}\right|^{2}=\sigma_{\text {inel }} / \sigma_{\text {el }}$. All parameters are then fixed by the value of the total and of the elastic nucleon-nucleon cross sections, namely by the imaginary part and by the modulus of $f_{11}$.

\section{The deuteron target}

In the simplest case of the deuteron target the expression of the the amplitude with a single rescattering is

$$
\begin{aligned}
\mathcal{A}^{(1)} & =-(1 / 2) \gamma^{2} x^{2} m \int d z_{1} d z_{1}^{\prime} d^{2} b \psi\left(b, z_{1}\right) i \Gamma(b) \psi\left(b, z_{1}^{\prime}\right) J\left(-z_{1}\right) J\left(z_{1}^{\prime}\right) e^{i \Delta\left(z_{1}^{\prime}-z_{1}\right)} \\
& =(1 / 2) \gamma^{2} x^{2} m \int d^{2} b i \Gamma(b)\left[X\left(b, x, Q^{2}\right)\right]^{2}
\end{aligned}
$$

where $b$ is the distance between the proton and the neutron in transverse space, $\psi(b, z)$ is the deuteron wave function and $\Gamma(b)$ the nucleon-nucleon profile function. To obtain the various contributions to the structure function one needs to consider the different discontinuities of the amplitude. To that purpose one may write:

$$
\begin{aligned}
X\left(b, x, Q^{2}\right) & =i \int d z \psi(b, z) J(-z) \exp (i \Delta z) \\
& =\int \frac{d^{3} k}{(2 \pi)^{3 / 2}} \phi(k) e^{i \mathbf{k}_{t} \mathbf{b}}\left[\frac{1}{k_{z}-\Delta-i 0}-\frac{1}{k_{z}-\Delta+\frac{1}{l}-i 0}\right]
\end{aligned}
$$

where $\phi(k)$ is the deuteron wave function in momentum space. The crosssection to produce a fast nucleon has two different contributions: from the cut of the amplitude $\Gamma$ and from the cut of the nucleon propagators. The sum of the two discontinuities from the cut nucleon propagators gives

$$
\operatorname{Disc}_{1} \mathcal{A}^{(1)}=i x^{2} m \gamma^{2} \int d^{2} b Y(b, x) \Re \mathrm{e}\left[i \Gamma(b) X\left(b, x, Q^{2}\right)\right]
$$


where

$$
Y(b, x)=\int \frac{d^{3} k}{(2 \pi)^{3 / 2}} \phi(k) e^{i \mathbf{k}_{t} \mathbf{b}_{2}} 2 \pi \delta\left(k_{z}-\Delta\right)
$$

and, in the Bjorken limit, is independent on $Q^{2}$. As for the discontinuity corresponding to a cut across the rescattering blob $\Gamma$, the contribution of the scattered nucleon to the inclusive structure function is obtained by taking only the elastic part of the unitarity sum over the intermediate states:

$$
\operatorname{Disc}_{2} \mathcal{A}^{(1)}=i(1 / 2) x^{2} m \gamma^{2} \int d^{2} b|\Gamma(b)|^{2}\left|X\left(b, x, Q^{2}\right)\right|^{2}
$$

The contribution to the inclusive deuteron structure function due to the nucleon rescattering in the final state, $F_{2}^{N / d,(1)}$, is given by the sum of these two discontinuities divided by $i$. At low energy, when no elastic channel is open and $\sigma_{\text {tot }}=\sigma_{e l}$, one obtains

$$
\begin{aligned}
F_{2}^{N / d,(1)} & =x^{2} m \gamma^{2} \int d^{2} b\left\{-2 \Im \mathrm{m} \Gamma(b)+\frac{|\Gamma(b)|^{2}}{2}\right\} \frac{[Y(b, x)]^{2}}{4} \\
& =x^{2} m \gamma^{2} \int d^{2} b\{-\Im \mathrm{m} \Gamma(b)\} \frac{[Y(b, x)]^{2}}{4}=2 \Im \mathrm{m} \mathcal{A}^{(1)}
\end{aligned}
$$

so that the only contributions to the imaginary part of the forward amplitude is given by the the two discontinuities (12) and (14), where only the elastic intermediate state is present. At higher energies the inelastic channels become important. The effect is to add further contributions to the imaginary part of the forward amplitude. As it may be seen by looking at the behavior of $X$, Eq.(11), as a function of the formation length $l$. The additional contributions give a small correction at low $Q^{2}$ (small $l$ ) while they tend to cancel completely the elastic contribution at large $Q^{2}$ (large $l$ ).

For a quantitative evaluation we write the deuteron structure function as

$$
F_{2}^{d}=F_{2}^{N / d,(0)}+F_{2}^{N / d,(1)}+F_{2}^{N^{*} / d,(1)}
$$

where $F_{2}^{N / d,(0)}$ is the expression in impulse approximation, the correction induced by FSI with a proton in the final state is $F_{2}^{N / d,(1)}$, and all other contributions to FSI are represented by $F_{2}^{N^{*} / d,(1)}$. In figure 2 we have plotted, as a function of $Q^{2}$, the ratios

$$
R_{N}\left(Q^{2}\right)=1+\left(\frac{F_{2}^{N / d,(1)}\left(x, Q^{2}\right)}{F_{2}^{N / d,(0)}\left(x, Q^{2}\right)}\right)_{x=1}
$$



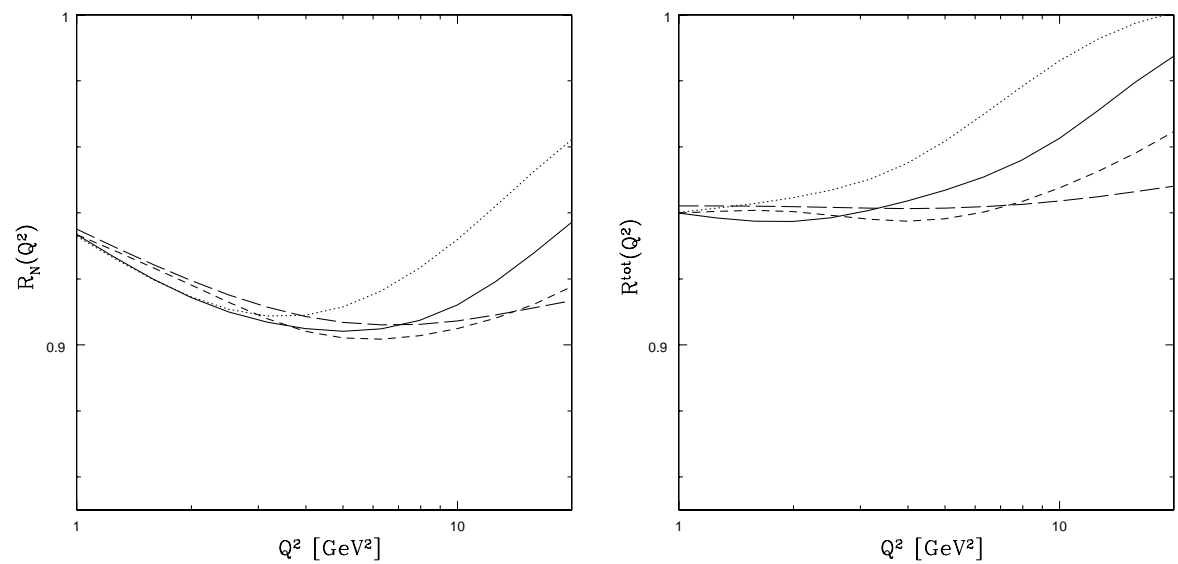

Figure 2. $R_{N}\left(Q^{2}\right)$ (eq. (17)) and of $R^{\text {tot }}\left(Q^{2}\right)$ (eq. (18)) at $x=1$ for the deuteron target with different choices of the excited nucleon mass: $m^{*}=1.44(\mathrm{GeV})$ dotted line, $m^{*}=1.8$ $(\mathrm{GeV})$ continuous line, $m^{*}=2.4(\mathrm{GeV})$ short-dashed line. The long-dashed line corresponds to the standard Glauber result, where no dependence of the amplitude on the virtuality of the external lines is taken into account.

and

$$
R^{t o t}\left(Q^{2}\right)=1+\left(\frac{F_{2}^{N / d,(1)}\left(x, Q^{2}\right)+F_{2}^{N^{*} / d,(1)}\left(x, Q^{2}\right)}{F_{2}^{N / d,(0)}\left(x, Q^{2}\right)}\right)_{x=1}
$$

at $x=1$, for the values $m^{*}=1.4,1.8,2.4 \mathrm{GeV}$ and for the pure Glauber case. $F_{2}^{N / d,(1)}$ is obtained summing the two discontinuities (12) and (14) while $F_{2}^{N / d,(1)}+F_{2}^{N^{*} / d,(1)}$ is evaluated by taking twice the imaginary part of (10). Looking at the continuous curve, corresponding to an excitation mass $m^{*}=$ $1.8 \mathrm{GeV}$, one observes that the threshold at which the FSI starts to vanish is practically the same in $R_{N}\left(Q^{2}\right)$ and in $R^{\text {tot }}\left(Q^{2}\right)$, the effect of FSI being sizably smaller in the latter quantity. The above formalism has been extended[4] to the so called cumulative region, i.e. at $x>1$, where a calculation within the Schroedinger and Glauber approaches shows that the latter might be inadequate [5]. 


\section{Transparency and momentum distributions in $\mathbf{A}\left(\mathbf{e}, \mathbf{e}^{\prime} \mathbf{p}\right) \mathrm{X}$ processes}

At relatively low $Q^{2}$ FSI effects are therefore well described by the Glauber approach. In this regime we have calculated the nuclear transparency and the distorted momentum distributions for ${ }^{4} \mathrm{He}$ [6] and complex nuclei [7]; for the former we have used a realistic four body wave function [8] whereas for the latter we have developed a number-conserving linked cluster expansion for the distorted one-body mixed density matrix with Glauber multiple scattering theory and with correlated nuclear wave functions containing realistic central and non-central correlations[7]. The linked cluster expansion includes final state interactions at all orders in the number of rescatterings while initial state correlations are taken into account at the lowest order. The results of the transparency for ${ }^{16} \mathrm{O}$ and ${ }^{40} \mathrm{Ca}$ are summarized in the tables, where $\left.i\right) T_{S M}$ is the value of transparency in the case of an uncorrelated nuclear wave function, ii) in $\Delta T_{F S I}^{S M}$ correlations are includes but FSI takes place with uncorrelated nucleons only (notice that in case of no FSI this term would be zero), iii) $\Delta T_{F S I}^{H}$ is the hole contribution (the struck nucleon is correlated), $\left.i v\right) \Delta T_{F S I}^{S, 1}$ and $\Delta T_{F S I}^{S, 2}$ are the spectator contributions (FSI takes place with a correlated

nucleon). In the linked expansion, as a consequence of the constraint of the conservation of the number of nucleons, one obtains in fact two spectator terms with opposite sign.
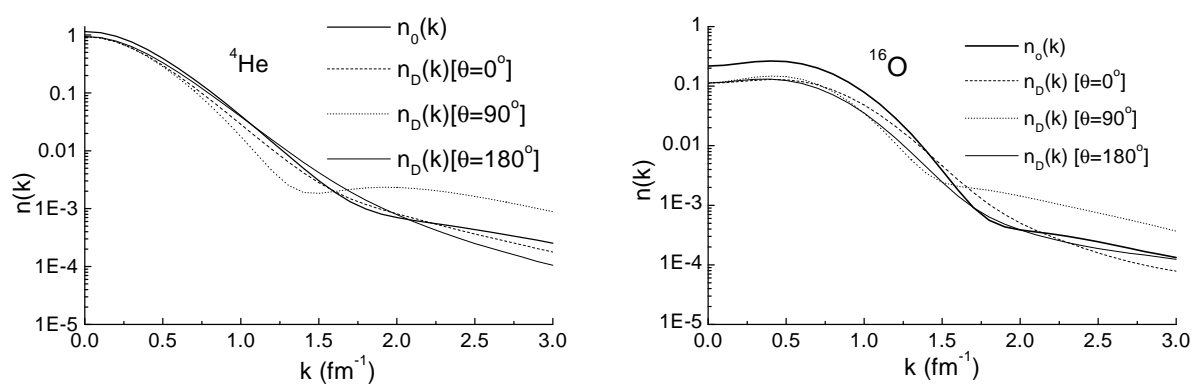

Figure 3. Distorted momentum distribution in ${ }^{4} \mathrm{He}$ and ${ }^{16} \mathrm{O}$. The full line represents the undistorted momentum distributions and the broken lines the distorted momentum distributions at parallel $\left(\Theta=0^{\circ}\right)$, antiparallel $\left(\Theta=180^{\circ}\right)$ and perpendicular $\left(\Theta=90^{\circ}\right)$ kinematics.

The distorted momentum distributions for ${ }^{4} \mathrm{He}$ and ${ }^{16} \mathrm{O}$ are shown in Fig.

cortona1: submitted to World Scientific on November 19, 2018 
Table 1. The nuclear transparency for ${ }^{16} \mathrm{O}$.

\begin{tabular}{lllllll}
\hline & $T_{S M}$ & $\Delta T_{F S I}^{S M}$ & $\Delta T_{F S I}^{H}$ & $\Delta T_{F S I}^{S, 1}$ & $\Delta T_{F S I}^{S, 2}$ & $T$ \\
\hline Central & 0.51 & 0.020 & 0.032 & -0.013 & 0.022 & 0.57 \\
Realistic & 0.51 & 0.003 & 0.009 & 0.001 & -0.001 & 0.52 \\
\hline
\end{tabular}

Table 2. The nuclear transparency for ${ }^{40} \mathrm{Ca}$.

\begin{tabular}{lllllll}
\hline & $T_{S M}$ & $\Delta T_{F S I}^{S M}$ & $\Delta T_{F S I}^{H}$ & $\Delta T_{F S I}^{S, 1}$ & $\Delta T_{F S I}^{S, 2}$ & $T$ \\
\hline Central & 0.41 & 0.020 & 0.028 & -0.011 & 0.023 & 0.47 \\
Realistic & 0.41 & 0.002 & 0.008 & -0.001 & 0.001 & 0.42 \\
\hline
\end{tabular}

3.

Our findings can be summarized as follows:

1. The effect of NN correlation on nuclear transparency $T$ amounts to $\sim$ $3 \%$. The results of the exact calculation for ${ }^{4} \mathrm{He}$ are consistent with those obtained with the cluster expansion for ${ }^{16} \mathrm{O}$ and ${ }^{40} \mathrm{Ca}$, indicating a rapid convergence of our number conserving cluster expansion. The small correction term is due to a cancellation between the short-range repulsive correlation and the intermediate-range attractive correlation terms, so that a calculation with short-range correlations only would give misleading conclusions.

2. The spectator effect is very small and thus there is no significant cancellation between the spectator and hole terms.

3. Double rescattering terms amount to $14 \%$ of the leading order term, namely the single rescattering contribution. Thus double rescatterings cannot be neglected.

4. As for the momentum distribution the FSI dominates the high momentum component in the direction perpendicular to the direction of the virtualphoton momentum, though its magnitude is reduced if one takes into account the tensor-type correlation which induce the $\mathrm{D}$-wave component in ${ }^{4} \mathrm{He}$. 
5. On the contrary, in the directions parallel and anti-parallel to the virtualphoton direction, the effect of FSI is sizably smaller.

\section{Final state interaction in exclusive A(e,e'p)B processes}

The formalism described in Section 1 has also been applied to the calculation of exclusive processes [10]. The results for the process ${ }^{3} H e\left(e, e^{\prime} p\right)^{3} H$ are presented in Figs. 4 and 5, where the effects from Glauber rescattering and Finite Formation Time are displayed for various values of $Q^{2}$. It can be seen that Glauber Final State Interactions has a large effect, in that it completely washes out the diffraction dip predicted by the Plane Wave Impulse Approximation; on the other hand side, they exhibit a very mild $Q^{2}$ dependence, unlike Finite Formation Time effects which strongly depend upon $Q^{2}$, in such a way that at $Q^{2} \simeq 20 \mathrm{GeV}^{2}$ the Plane Wave Impulse Approximation result is almost completely recovered.

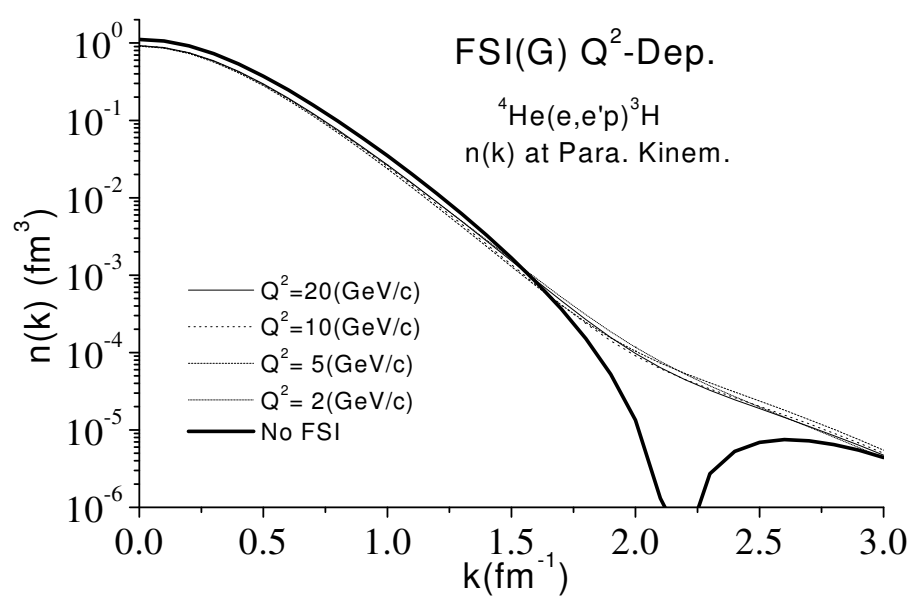

Figure 4. The effects from Glauber rescattering on the distorted momentum distributions in the exclusive process ${ }^{4} \mathrm{He}\left(e, e^{\prime} p\right)^{3} \mathrm{H}$ at parallel kinematics. The full curve represents the Plane Wave Impulse Approximation result, whereas the broken lines include the Glauber rescattering at various values of $Q^{2}$

cortona1: submitted to World Scientific on November 19, 2018 


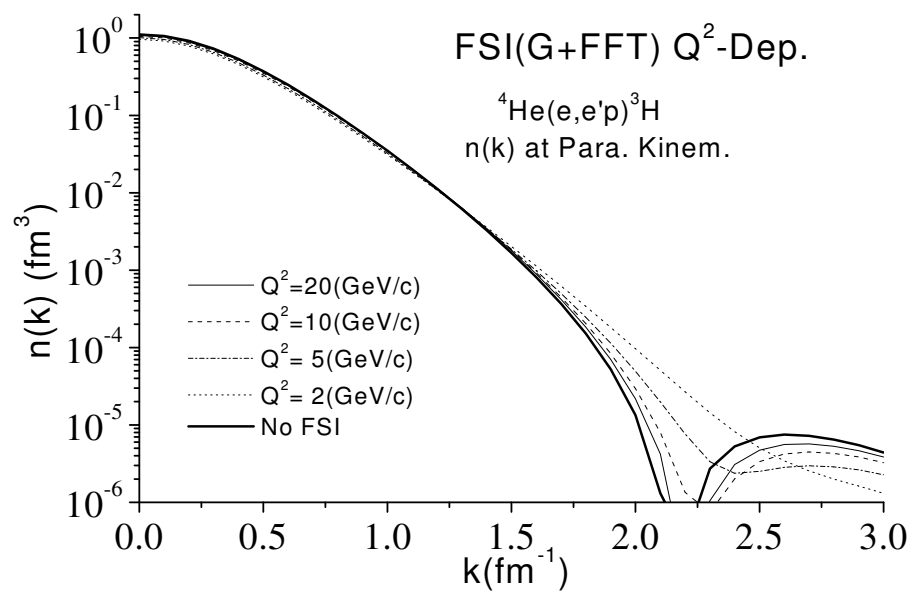

Figure 5. Same as Figure 4, with Glauber rescattering plus Finite Formation Time effects.

\section{References}

[1] V.N.Gribov, Sov. Phys. JETP 29, 483 (1969); 30 (1970) 709; L. Bertocchi, Nuovo Cimento 11A, 45 (1972);

[2] M.A.Braun, C.Ciofi degli Atti and D. Treleani Phys. Rev. C62, 034606 (2000);

[3] L. Frankfurt, W.R. Greenberg, G.A. Miller and M. Strikman, Phys. Rev. C46, 2547 (1992);

[4] M. Braun, C. Ciofi degli Atti, L. Kaptari and D. Treleani, to appear;

[5] C. Ciofi degli Atti, L. Kaptari, and D. Treleani, Phys. Rev., to appear;

[6] H. Morita, C. Ciofi degli Atti and D. Treleani, Phys. Rev., C60, 034603 (1999);

[7] C. Ciofi degli Atti and D. Treleani, Phys. Rev., C60, 024602 (1999);

[8] M. Alvioli, C. Ciofi degli Atti H. Morita and D. Treleani, to appear;

[9] H.Morita, Y.Akaishi, O.Endo and H.Tanaka Prog.Theor. Phys. 78, 1117 (1987);

[10] M. Braun, C. Ciofi degli Atti, H. Morita and D. Treleani, to appear. 